\title{
Le nouveau mécanisme de sauvegarde des entreprises en difficulté: une vraie "bouée de sauvetage" pour les entreprises naufragées?
}

The new safeguard mechanism for companies in difficulty: a real "lifeline" for wrecked companies?

\author{
NAHID LYAZAMI* \\ Professeure de Droit des Affaires Habilitée à diriger des recherches doctorales (HDR) \\ Université Abdelmalek Assaidi Tanger (Maroc) \\ nahidlyazami@hotmail.com \\ https://orcid.org/0000-0002-9585-5289
}

Résumén: Le législateur Marocain soucieux de préserver des entités viables et saines, avait envisagé à travers la nouvelle loi 73-17 un nouveau procédé de sauvetage des entreprises en difficultés juridiques, économiques, financières et sociales.

Ce nouveau mécanisme fortement inspiré de la législation française (loi de sauvegarde) tendait essentiellement à venir en aide aux entreprises qui éprouvent des difficultés insurmontables sans pour autant atteindre la phase de la cessation de paiement.

Abstract: The Moroccan legislator, concerned with preserving viable and healthy entities, had envisaged through the new law 73-17 a new process for rescuing companies in legal, economic, financial and social difficulties.

Cómo citar este trabajo: LYAZAMI, Nahid, "Le nouveau mécanisme de sauvegarde des entreprises en difficulté: Une vraie 'bouée de sauvetage' pour les entreprises naufragées?”, Revista de Estudios Jurídicos y Criminológicos, ISSN-e: 2660-7964, n. ${ }^{\circ}$ 2, Universidad de Cádiz, 2020, pp. 13-39, DOI: https://doi.org/10.25267/REJUCRIM.2020.i2.02

\footnotetext{
* Docteur en Droit.
} 
This new mechanism, strongly inspired by French legislation (safeguard law), essentially tended to come to the aid of companies that are experiencing insurmountable difficulties without however reaching the phase of insolvency.

Palabras claves: detección temprana, dificultades para empresas, prevención, procedimiento de respaldo.

Keywords: early detection, difficulties for companies, prevention, safeguard mechanism.

Sommaire: 1. INTRODUCTION. 2. LA PREVENTION-DETECTION PAR LE BIAIS DE LA PROCEDURE DE SAUVEGARDE. 2.1. le recadrage des critères d'accès à la procédure de sauvegarde. 2.1.A. Faits justificatifs. 2.1.B. Caractérisation in concreto des déboires financiers rencontrés par le demandeur. 2.2. les effets subséquents à l'adoption du plan de sauvegarde. 2.2.A. L'éviction du dirigeant n'est plus envisageable. 2.2.B. Le procédé de déclaration des créances: un passage obligé? 3. QUELLE ATTRACTIVITE POUR LES PARTENAIRES DU DEBITEUR? 3.1. La sauvegarde entre emballement des uns et désengouement des autres. 3.1.A. La constitution de comités des créanciers: une méthode de concertation efficiente? 3.1.B. La préservation du mécanisme de suspension provisoire des poursuites individuelles. 3.2. La qualification juridique du nouveau mécanisme de sauvetage. 3.2.A. La sauvegarde: une procédure amiable, collective ou hybride? 3.2.B. Le caractère "ambivalent" de la nouvelle procédure de sauvegarde. 4. EPILOGUE. 5. BIBIOLGRAPHIE.

\section{INTRODUCTION}

Au-delà de l'instauration d'une procédure de conciliation, une procédure dite de "sauvegarde" a été introduite récemment en doit Marocain, tout en rénovant totalement le droit des entreprises en difficulté. On se réfère désormais à la loi 73-17 du Dahir n¹-18-26 du 19 Avril 2018 publiée au Bulletin Officiel nº6732 du 6 décembre 2018 ${ }^{1}$, ci-après la loi 73-17 relative aux difficultés de l'entreprise.

Cette nouvelle loi, qui complète utilement "la boite à outils "2, du livre V du code de commerce, constitue une procédure à mi-chemin, entre la procédure préventive de conciliation et les procédures curatives de traitement.

\footnotetext{
${ }^{1}$ Consultez le secrétait général du governement.www.sgg.gov.ma.

${ }^{2}$ ROUSSEL GALLE, F., "La procédure de sauvegarde : quand et pourquoi se mettre sous la protection de la justice”, Jurisclasseur périodique, Edition 2006, p. 2437.
} 
L'arsenal législatif Marocain a fait l'objet d'un dépoussiérage de ses dispositions législatives jugées surannés et entachées d'anachronisme, et ce par l'abrogation du livre $\mathrm{V}$ de la loi 15-95 formant le code de commerce relatif aux difficultés de l'entreprise et son remplacement par la loi 73-17. Un texte de refonte qui est resté en vielleuse pendant bien longtemps.

Le législateur a pu enfin mettre à la disposition des entités en difficulté un procédé susceptible d'assurer la préservation des entreprises saines et viables éludant ainsi l'aggravation de leurs difficultés, voire leur disparition le cas échéant.

Une nouvelle architecture du droit des entreprises en difficulté a pu voir le jour, une nouvelle version qui tend essentiellement à satisfaire à tous les intervenants, faisant en sorte de conjuguer plusieurs impératifs sans bafouer les droits des uns et privilégier les intérêts des autres.

L'adoption de la loi fut annoncée de "but en blanc", que plusieurs praticiens l'ont estimée ainsi brusque et précipitée, et n'ont pas hésité de montrer leur sidération quant aux circonstances de son entrée en vigueur.

Si certaines lois sont caractérisées par le repli sur soi, la loi Marocaine plus précisément le droit des procédures collectives, est très marqué par son ouverture sur le droit comparé Français ${ }^{4}$, qui constitue une source d'imprégnation privilégiée.

La procédure de prévention des difficultés des entreprises est opportune face aux éclatements récurrents du contentieux commercial et économique. Les évolutions constatées afin

\footnotetext{
${ }^{3}$ Loi n¹995-15 du Dahir n¹-96-83 du 1 er Aout 1996. Bulletin Officiel n4418 du 3 octobre 1996. Cette Loi avait remplacé l'ancien code terrestre de 1913 qui traite la faillite du débiteur d'entreprise en difficulté. Ce code "très rigoureux" fût une loi sanctionnatrice, coercitive visant à éjecter le débiteur du monde des affaires en raison de son insolvabilité, peu importe qu'il soit malhonnête ou malchanceux et victime de conjonctures qui lui dépassent. Par contre la loi 15-95 et la 73-17 permettaient au chef d'entreprise malheureux de pouvoir bénéficier de la règle de la "seconde chance" afin d'éviter au maximum le recours aux procédures contentieuses de redressement, ou la de liquidation judiciaire le cas échéant.
}

${ }^{4}$ Loi n. ${ }^{\circ} 84-148$ du 1 mars 1984 relative à la prévention et au règlement amiable des difficultés des entreprises.

Loi n. ${ }^{\circ} 85-98$ du 25 janvier 1985 relative au redressement et à la liquidation judiciaires des entreprises.

Loi n. ${ }^{\circ}$ 94-475 du 10 juin 1994 relative à la prévention et au traitement des difficultés des entreprises

Loi n. ${ }^{\circ}$ 2005-845 du 26 juillet 2005 de sauvegarde des entreprises, entrée en vigueur le 1er janvier 2006, et son décret d'application n²005-1677 du 28 décembre 2005 favorisant le sauvetage, la prévention et la négociation, également applicable aux professions libérales et indépendantes.

Le droit des entreprises en difficulté français, est un droit en " perpétuel chantier " comme en attestent les réformes successives de 2008, 2010, 2011, 2012, 2014, 2015,2016.

La loi de régulation bancaire et financière, adoptée définitivement par l'Assemblée nationale le 11 octobre 2010, a été publiée au Journal Officiel du 23 octobre 2010 créant la procédure de sauvegarde accélérée et sa déclinaison ou sa variante qui est sauvegarde financière accélérée.

Loi n 2019-486 du 22 mai 2019 relative à la croissance et la transformation des entreprises. Journal officiel de république Française (ci-après JORF) nº 0119 du 23 Mai 2019. 
d'harmoniser le texte législatif et les pratiques attestent de l'énergie manifestée par le législateur Marocain. Certes, des manquements demeurent toujours, mais les procédures mises à la disposition des hommes d'affaires sont d'un intérêt indéniable. Car, puisque le mal des entreprises ne connait pas de grève, il faut donc trouver la relève pour le juguler.

Si pour certains commentateurs de cette nouvelle loi, la mesure baigne dans un climat de bienveillance car elle permettra aux entreprises de remonter la pente et de corriger les irrégularités de leurs trésoreries, par la reconstruction de l'actif patrimonial et la restructuration de l'endettement, pour certains ténors du droit des procédures collectives l'avènement de cette loi n'a pas reçu l'engouement tant espéré 5 .

Si le dicton populaire disait “on ne mesure pas les hommes à la toise, c'est par le degré de mérite qu'on les apprécie", ce même raisonnement doit être adopté. On ne peut pas juger du degré d'applicabilité et d'efficience de la nouvelle loi 73-17, tant qu'on n'a pas réalisé des avancées sophistiquées en matière de sauvetage des entreprises en difficulté.

Après plus de deux ans depuis son entrée en vigueur, déjà un constat global a pu être tiré concernant les résultats et les perspectives à l'avenir qui, à notre sens, constituent des conclusions et des jugements hâtifs et précipités condamnant le dispositif en vigueur, car des législations comparées ont pu juger de son efficacité et sa capacité à rendre à meilleure fortune les entreprises qui souffrent de difficulté de règlement de leurs dettes.

Selon le garde des sceaux Français à l'époque en fonction. CLEMENT "le principe même de la sauvegarde, pivot de ce droit profondément rénové, nous fait passer de l'ère du dépôt de bilan à celle de la demande de protection judiciaire". La première était synonyme d'échec, la seconde sera synonyme d'espoir'.

A travers cette étude, notre ambition sera de dévoiler est ce que cette procédure constitue vraiment une panacée pour les entreprises victimes de conjonctures qui dépassent leurs capacités de rebondissement, ou bien c'est une sorte de frénésie législative ${ }^{7}$ commandée par le souci d'être toujours au diapason des législations étrangères, qui sont au palmarès en matière d'innovations législatives ${ }^{8}$. On va donner un focus sur les critères d'accès à cette procédure et les effets subséquents.

\footnotetext{
${ }^{5}$ LYAZAMI, N., "plus de vingt ans d'application du droit préventif de difficulté de l'entreprise : un bilan mitigé", Revue Marocaine d'administration locale et de développement, $\mathrm{n}^{\circ} 148$, septembre-octobre, 2019, p. 110 et suiv.

${ }^{6}$ CLEMENT, P., “Avant-propos du garde des Sceaux. Ministre de la justice”: Gaz. Pal. 8 sept. 2005, p. 3.

${ }^{7}$ LYAZAMI, N., "plus de vingt ans d'application du droit préventif des difficultés de l'entreprise: un bilan mitigé" Revue Remald, n. ${ }^{\circ} 141$. Juillet-Août 2018, p. 120.

8 Directive (UE) 2019/1023 du parlement européen et du conseil du 20 juin 2019 relative aux cadres de restructuration préventive, à la remise de dettes et aux déchéances, et aux mesures à prendre pour augmenter l'efficacité des procédures en matière de restructuration, d'insolvabilité et de remise de dettes, et modifiant la directive (UE) 2017/1132 (directive sur la restructuration et l'insolvabilité). Initiée par la loi Pacte $n^{\circ}$ 2019-486 du
} 
On sera tenté également de dresser un bilan provisoire sur son degré d'application même s'il est un peu précoce pour tirer des conclusions, car ce nouveau-né législatif vient de souffler sa deuxième bougie, il n'y a pas bien longtemps. Après l'écoulement de deux années quel constat peut-on en tirer?

Notre analyse portera sur la comparaison avec le droit Français, gisement inestimable pour le législateur Marocain. En dénichant les points de ressemblance ou de divergence éventuellement.

\section{LA PRÉVENTION-DÉTECTION PAR LE BIAIS DE LA PROCÉDURE DE SAUVEGARDE}

Si le droit Français depuis $2005^{9}$ et jusqu'aujourd'hui à travers la promulgation de la loi pacte ${ }^{10}$ a pu réaliser des avancées très spectaculaires en matière de sauvetage des entreprises, leurs consœurs Marocaines sont restées privées de toutes ces prérogatives jusqu'au 2018.

La sauvegarde était adoptée en 2005 en France, ce n'est qu'après 13 ans que le législateur marocain a pris conscience quant à la possibilité de cette procédure de sauver ou de restructurer des entreprises qui remplissent les critères requis.

Dire que la procédure de sauvegarde est plus attractive n'est pas hasardeux. L'entrée en vigueur de l'ordonnance du 18 décembre 2008 complétée par un décret d'application le 12 février 2009 a bien marqué les esprits. C'était l'occasion pour aborder des retouches et des mesures de simplification des textes, mais sans que cela produise un remaniement de l'architecture de la loi de sauvegarde du 26 juillet 2005. La réforme introduite a rendu la procédure de sauvegarde plus efficiente pour le débiteur.

Le droit Français a fait des avancées très sophistiquées en matière de procédure de sauvegarde, afin d'augmenter son efficacité et de sauvegarder et côtoyer les entreprises viables quand elles passent par des moments de difficulté de trésorerie.

Il convient de signaler que cette même loi de sauvegarde a été modifiée sur deux points très intéressants. D'abord les comités des créanciers, introduits par la loi du 26 juillet 2005 puis réformés par l'ordonnance du 18 décembre 2008.

22 mai 2019 relative à la croissance et à la transformation des entreprises. Journal Officiel de l'union européenne.26 Juin 2019.

${ }^{9}$ La Loi n²005-845 du 26 juillet 2005 de sauvegarde des entreprises, entrée en vigueur ,1er janvier 2006, et son décret d'application n²005-1677 du 28 Décembre 2005. Livre VI du code de commerce français.

${ }^{10}$ Loi pacte $\mathrm{n}^{\circ} 2019-486$ du 22 mai 2019 relative à la croissance et la transformation des entreprises qui favorise la culture du rebond et qui offre une seconde chance aux entreprises victimes de difficultés conjoncturelles afin de leur permettre de rebondir. Cette loi à travers "la procédure du rétablissement professionnel du chef d'entreprise" lui confère la possibilité de bénéficier d'un effacement de ses dettes. 
Ensuite, ce qui est le plus marquant, c'est la création d'une sous-procédure dite sauvegarde financière accélérée (ci-après SFA), dont le but est la restructuration de l'endettement, réservée à de grandes entreprises remplissant des conditions bien spécifiques et déterminées. Cet outil permet de favoriser l'adoption d'un plan de sauvegarde et tente à mettre terme aux oppositions des créanciers minoritaires, récalcitrants qui refusent de faire des concessions.

FRANCOIS XAVIER ${ }^{11}$ considérait cette procédure de SFA comme une procédure collective au sens "qu'elle emporte une saisie collective du patrimoine du débiteur", tandis que SAINTHALARY HOUIN ${ }^{12}$ estimait qu'elle est une procédure "élitiste” et "instrumentalisée".

Toujours dans la perspective de maintenir des entreprises saines et solvables, le législateur Français via l'ordonnance du 12 mars 2014 a notamment créé la procédure de sauvegarde accélérée pour les grandes entreprises préalablement engagées dans une procédure de conciliation.

En s'imprégnant du législateur Français, et pour être au palmarès des législations avancées, le Maroc tout en bousculant totalement le droit des entreprises en difficulté, avait prévu par la loi $73-17^{13}$ le procédé de sauvegarde tendant essentiellement à augmenter l'efficacité et à assurer l'ancrage optimal des mesures préventives anticipatives mais également à sauvegarder et côtoyer les entreprises viables quand elles passent par des moments de difficulté de trésorerie sporadiques qui ne nécessitaient pas vraiment des mesures curatives.

Le livre V du code de commerce Marocain traitant les procédures de difficultés de l'entreprise offre une large palette de procédés ou procédures dites soft visant précisément la préservation des entreprises saines, en évitant qu'elles dérapent vers les procédures de traitement dont l'issue n'est pas toujours bienveillante.

L'éclosion de la nouvelle procédure de sauvegarde a suscité l'engouement des uns comme elle a été jugée comme inutile par les autres puisqu'elle présente des traits de similitude avec la conciliation, et son avènement ne va que rendre les justiciables encore plus perplexes quant au choix qu'ils doivent faire.

Si on pourra tirer des leçons après plus de deux ans d'application de la procédure de sauvegarde au Maroc, on est incapable de parler de sa variante qui est la sauvegarde financière accélérée car elle est méconnue par le droit Marocain.

\footnotetext{
${ }^{11}$ FRANCOIS-XAVIER, L., Colloque Que reste-il des principes traditionnels des procédures collectives face au Morcellement du traitement de la défaillance”, 25 novembre 2011.université paris ouest Nanterre la défense.

${ }^{12}$ SAINT-HALARY HOUIN, C., "Les leçons de cinq d'application de la loi de sauvegarde", Montpellier I.la sauvegarde financière accélérée, p. 32 et suive.

${ }^{13}$ La procédure de sauvegarde est régie par le TITRE III du LIVRE V de la loi 73-17 relative aux procédures de difficultés de l'entreprise par les articles 560 à 574.
} 
La nouvelle procédure de sauvegarde vise particulièrement à surmonter les difficultés de l'entreprise, pour assurer la poursuite de son activité, l'apurement du passif et le maintien des emplois $^{14}$.

Parmi les finalités de l'adoption de cette nouvelle loi 17-73 figure le fait d'assurer une attractivité pour les procédures amiables qui ont beaucoup perdu en notoriété en raison de plusieurs facteurs intrinsèques ${ }^{15}$.

Non seulement l'anticipation est le fondement de cette loi et la prévention est sa leitmotiv, mais encore, le chef d'entreprise débiteur n'est pas dessaisi de ses prérogatives; il poursuit son activité, éventuellement surveillé par la justice. C'est un autre aspect non négligeable du caractère novateur de la loi.

A quoi consiste la nouvelle procédure de sauvegarde? Quels sont ses conditions d'ouverture? Et quel est le sort des créanciers et du débiteur après l'adoption d'un plan de sauvegarde?

\subsection{Le recadrage des critères d'accès à la procédure de sauvegarde}

La volonté du législateur Marocain est de faire bénéficier le débiteur en difficulté, avant de se retrouver dans la cessation de paiement, d'une protection de la justice, sous l'égide de laquelle, il va s'abriter contre les poursuites de ses créanciers, jusqu'à ce qu'il parvienne à élaborer un accord dit "plan de sauvegarde" avec ces derniers.

Ces critères de recadrage permettent de veiller à ce que cette procédure ne soit pas ouverte à mauvais escient, ou utiliser de façon incongrue. Le but c'est d'obtenir une "réorganisation"16 de l'entreprise, le maintien de l'emploi, et l'apurement du passif ${ }^{17}$.

Le législateur n'a pas voulu laisser au débiteur toute la latitude pour demander le bénéfice de la procédure de sauvegarde, il redoutait l'abus du débiteur à vouloir saisir le juge dans le seul but de limiter ou de nuire aux intérêts légitimes de ses créanciers. Le législateur a voulu éviter l'utilisation détournée ou démesurée de cette procédure.

\footnotetext{
${ }^{14}$ Art. 560 de la loi 73-17 relative aux difficultés de l'entreprise.

${ }^{15}$ LYAZAMI, N., "la Prévention des difficultés des entreprises : approche comparative entre le Droit Marocain et le Droit Français", Thèse de doctorat en Droit privé, Université du Sud de Toulon-Var. 4 juin 2013, p. 260.

${ }^{16}$ Le choix du terme "réorganisation", laisse entendre que l'objectif n'est pas de céder ou liquider l'entreprise, mais de poursuivre l'activité, en procédant aux mesures nécessaires pour assurer sa pérennité. Le choix du terme de "sauvegarde", traduit une approche plus optimiste, de connotation positive sur le sort de l'entreprise, s'éloignant de l'échec qui caractérise trop souvent le redressement judiciaire.

${ }^{17}$ L'art. 560 du livre V de la loi 73-17 relative aux difficultés des entreprises.
} 


\subsection{A. faits justificatifs}

Les faits justifiants la demande d'ouverture de la sauvegarde et selon les termes de l'art. 561 de la loi 73-17 sont "la procédure de sauvegarde peut être ouverte à toute entreprise commerciale ${ }^{18}$ qui sans être en cessation de_paiement éprouve des difficultés ${ }^{19}$ qu'elle n'est pas en mesure de surmonter de nature à conduire dans un délai proche à la cessation des paiements" ${ }^{\text {20 }}$.

Si le législateur Marocain, évoque la précision de "nature à conduire à conduire à la cessation de paiement", son homologue Français a écarté cette perspective ${ }^{21}$.

Généralement c'est la situation financière qui incite les juges à accepter d'ouvrir la procédure de sauvegarde, mais dans une résolution jurisprudentielle Française ${ }^{22}$, c'est le conflit social qui a été mis en évidence. La société était encore bénéficiaire, mais le poids de ses emprunts était singulièrement important et personne dans l'entreprise n'était en mesure de déterminer sa situation financière exacte.

Il y avait des signes prémonitoires de difficultés sociales très avérées comme :

- L’absence d'une vraie direction (directeur de la société est présent de façon intermittente);

- La multiplication des conflits familiaux et sociaux;

- Blocage majeur et mouvements de grève récurrents;

- L'actionnaire majoritaire tergiversait sur l'option de la cession de l'entreprise;

\footnotetext{
${ }^{18}$ Son homologue Français avait élargi la palette des personnes bénéficiaires de la sauvegarde. L'art. 620-2 du code de commerce français dispose : "La procédure de sauvegarde est applicable à toute personne exerçant une activité commerciale, artisanale ou une activité agricole définie à l'art. L. 311-1 du code rural et de la pêche maritime et, à toute autre personne physique exerçant une activité professionnelle indépendante, y compris une profession libérale soumise à un statut législatif ou réglementaire ou dont le titre est protégé, ainsi qu'à toute personne morale de droit privé".
}

${ }^{19}$ A préciser que la loi Marocaine, ne s'est pas exprimée sur la nature de ces difficultés, un terme général qui peut englober, celles économiques, financières, juridiques et sociales voire politiques.

${ }^{20}$ Art. L 620-1 du code de commerce Français dispose "Il est institué une procédure de sauvegarde ouverte sur demande d'un débiteur mentionné à l'art. L. 620-2 qui, sans être en cessation des paiements, justifie de difficultés qu'il n'est pas en mesure de surmonter. Cette procédure est destinée à faciliter la réorganisation de l'entreprise afin de permettre la poursuite de l'activité économique, le maintien de l'emploi et l'apurement du passif'.

${ }^{21}$ Depuis l'ordonnance de 18 décembre 2008, le texte est rédigé comme suit "il est institué une procédure de sauvegarde ouverte sur demande d'un débiteur, qui sans être en cessation de paiement, justifie de difficulté qu'il n'est pas en mesure de surmonter".

${ }^{22}$ Les tribunaux ont été conduits à accorder l'ouverture de la procédure de sauvegarde à une entreprise employant 170 salariés lorsqu'ils ont déduit qu'elle justifiait des difficultés qu'elle n'est pas en mesure de surmonter, de nature à conduire à la cessation des paiements. C'est le cas dans un jugement du TGI.ch.com. Bressuire, 19 décembre 2007. n07-4. 
- L’intention du président de présenter sa démission.

En France, cette notion de cessation de paiement a été éludée par la réforme de l'ordonnance de 18 décembre $2008^{23}$, qui a retranché l'exigence d'une perspective de cessation de paiement. Le but de cette réécriture était de rendre moins rigide et d'assouplir les conditions d'ouverture de la sauvegarde, en déconnectant les difficultés justifiant cette ouverture de la cessation de paiement.

L'existence de "difficultés qui ne peuvent être surmontées" signifie que le débiteur n'était pas en mesure de vaincre seul ses difficultés sans un plan. Les tribunaux ont tendance à recourir de plus en plus à des éléments subjectifs, moins comptables et plus humains, pour apprécier la situation du débiteur. Il n'est plus question de limiter les difficultés à “des difficultés juridiques, économiques, et financières" et c'est cette même vision que partageait certains auteurs, comme CHAPUT "la démonstration n'en sera pas uniquement comptable, la subjectivité est à considérer : maladie, âge, découragement du débiteur" 24 .

Tant en droit Français qu'en droit Marocain, Deux conditions sine qua none doivent être réunies de façon cumulative et non pas alternative, la première condition est négative est celle de l'absence de l'état de cessation de paiement, ci-après CP la deuxième condition est positive il s'agit de l'existence des difficultés insurmontables pouvant conduire à la cessation des paiements dans un futur proche.

La doctrine française ${ }^{25}$ estime que la cessation de paiement en tant que notion reflétant l'insolvabilité du débiteur doit être déconnectée de la phase de prévention, d'anticipation et de rebondissement, car elle crée chez les partenaires de l'entreprise un attentisme excessif, un scepticisme accru et dénature le caractère anticipatif de la prévention.

\subsection{B. Caractérisation in concreto des déboires financiers rencontrés par le demandeur}

La loi Marocaine impose au débiteur de convaincre le tribunal qu'il se trouve dans une situation insurmontable. Les raisons qui justifient cette position c'est d'éviter que le débiteur profite des avantages de cette procédure au détriment des autres créanciers, car la sauvegarde présente pour ces derniers un inconvénient non négligeable.

\footnotetext{
${ }^{23}$ Ordonnance $\mathrm{n}^{\circ}$ 2008-1345 du 18 décembre 2008 portant réforme du droit des entreprises en difficulté. Journal officiel de la république française ci -après (JORF).n0295 du 19 décembre 2008.

${ }^{24}$ CHAPUT, Y., "Une nouvelle architecture du droit français, des procédures collectives", Jurisclasseur périodique. Ci-après", JCP, 2005, p. 184.

${ }^{25}$ VALLANSAN, J., “Que reste-t-il de la cessation des paiements?”, Revue des Procédures Collectives, dossier 13, 2012; FAURY D., "La notion de cessation des paiements et la loi dite de sauvegarde des entreprises", Gazette du Palais, 23 janv. 2007, nG2924, p. 8.
} 
Elle entraine un arrêt des poursuites et donc, le créancier ne peut plus agir en paiement, contre le débiteur, qui bénéficiera d'une période pendant laquelle, il pourra reconstituer sa trésorerie. Ensuite, si le créancier omet de déclarer sa créance dans les délais légaux, il perd tout droit à la répartition et s'il refuse de consentir des délais de paiement ou des remises de dettes, il sera subordonné à des délais de paiements imposés dans le cadre du plan de sauvegarde.

Le créancier qui s'abstient à déclarer sa créance dans les délais impartis sera frappé de Forclusion. Cependant, une action en relevé de forclusion peut être déclenchée s'il arrive à prouver que son inertie était due à une force majeure ${ }^{26}$.

La demande d'ouverture de la procédure de sauvegarde doit être faite par le chef d'entreprise en difficulté auprès du tribunal territorialement compétent, sa demande doit être déposée au service du secrétariat greffe tout en exposant la nature des difficultés ${ }^{27}$ qui vont compromettre la continuité de l'exploitation de son entreprise comme il doit faire parvenir au tribunal (preuve à l'appui) tout document qui atteste de l'existence et de l'aggravation de ces difficultés dans un futur proche.

Le débiteur doit joindre à sa demande d'ouverture de sauvegarde, un "projet de plan de procédure de sauvegarde" qui doit énoncer les mesures nécessaires pour préserver l'activité de l'entreprise et définir les modalités de règlement du passif, ainsi que les garanties disponibles et proposées pour financer le dit plan et assurer son exécution.

L'existence de "difficultés" doit être confortée par des chiffres financiers et comptables, d'où le rôle primordial des commissaires aux comptes-experts comptables.

L'acceptation ou le refus d'adoption d'un plan de sauvegarde revient au tribunal de commerce qui avant de trancher pourra valablement consulter toutes les entités (Caisse Nationale de la Sécurité Sociale- service de fiscalité-trésorerie du Royaume-les banques...) ${ }^{28}$ qui peuvent lui fournir des informations plus amples sur la situation économique, financière et sociale de l'entreprise boiteuse comme il peut mener une expertise dans ce sens en faisant appel à un expert.

Le tribunal toujours ; à qui revient le dernier mot, est tenu de convoquer personnellement le chef d'entreprise à huit clos (en chambre de conseil) afin d'écouter ses propos et ce dans un délai de 15 à partir du dépôt de la demande d'ouverture de la sauvegarde.

\footnotetext{
${ }^{26}$ Arrêt de la cour de cassation, ch.com, Rabat, $n^{\circ} 146-878-3-2-2003$, Revue gazette des tribunaux du Maroc, $\mathrm{n}^{\circ} 108$, p. 49.

${ }^{27}$ Art. 561 du code commerce Marocain, 2 éme alinéa.

${ }^{28}$ Ces entités ne peuvent pas se retrancher derrière le secret professionnel et s'abstenir de fournir les informations nécessaires, toute disposition contraire est réputée non avenue (art. 563 du code de commerce Marocain).
} 
Ajoutant également, que la situation de l'entreprise doit être appréciée en elle-même, sans que soit prises en compte les capacités financières du groupe auquel elle appartient ; ce qui signifie que les difficultés doivent être insurmontables pour le débiteur, avec ses seules ressources et sans que l'existence ou l'imminence de poursuites de la part des créanciers soient exigées.

Les juges doivent apprécier ces difficultés in concreto, en se plaçant au jour où ils statuent. C'est la date à laquelle il est procédé à l'ouverture de la procédure, ce n'est pas la situation à la date de la saisine qui importe, car il a pu être constaté qu'au jour de la demande, les éléments n'étaient pas à eux seuls suffisants pour obtenir l'ouverture de la sauvegarde ; mais que c'est leur appréciation au jour du jugement qui a permis au juge d'être convaincu de la nécessité impérieuse de faire bénéficier le débiteur d'un plan de sauvegarde .

La cour d'appel doit se situer au jour du jugement pour apprécier le bien-fondé de l'ouverture et non pas au jour où elle statue. Car il se peut qu'entre le jugement et l'audience de la cour d'appel, un événement ait lieu et mette fin aux difficultés. Dans cette hypothèse, la cour de cassation refuse à la cour d'appel de réformer le jugement mais elle pourra convertir la sauvegarde en redressement judiciaire en cas de constatation d'une cessation de paiement.

Art. 564 de la loi 73-17 prévoit la conversion de la procédure de sauvegarde en une procédure de traitement des difficultés s'il s'est avéré que le jour du jugement d'ouverture de la procédure l'entreprise était déjà sombrée dans la cessation de paiement, devant cette hypothèse le tribunal doit fixer la date de cessation de paiement qui ne peut être antérieure à 18 mois de la date du jugement d'ouverture art. 713 de la nouvelle loi 73-17.

Si l'ouverture de la sauvegarde suppose l'absence de l'état de cessation de paiement du débiteur, il serait anodin de préserver un plan de sauvegarde pour une entreprise qui cesse de payer ses dettes, l'immixtion de la notion de cessation de paiement (ci-après $\mathrm{CP}$ ) à mi-chemin entraine inéluctablement la substitution de la sauvegarde par un redressement ou une liquidation selon la situation de l'entreprise moribonde. On peut dire que la notion de Cessation des Paiements est une ligne séparatrice entre l'amiable et le contentieux ${ }^{29}$.

\subsection{Les effets subséquents à l'adoption du plan de sauvegarde}

La nouvelle procédure de sauvegarde "revalorise la situation du débiteur", elle lui permet de se prémunir contre les poursuites de ses créanciers. Ce dispositif permet au dirigeant de rester davantage maitre de la procédure, sous le contrôle du tribunal de commerce, parce qu'il n'est pas mis en concurrence avec des tiers qui pourraient proposer le rachat de l'entreprise. Quand l'entreprise est en sauvegarde, cela signifie qu'elle n'est pas encore défaillante ; il n'y a à ce stade aucune raison d'empêcher le dirigeant de gérer l'entreprise.

\footnotetext{
${ }^{29}$ LYAZAMI, N., "la Prévention des difficultés des entreprises : approche comparative entre le Droit Marocain et le Droit Français", Thèse de doctorat en Droit privé, Université du Sud de Toulon,-Var. 4 juin 2013.
} 
L'ouverture de la procédure de sauvegarde entraine la suspension provisoire des poursuites individuelles de la part des créanciers, visant à geler le passif patrimonial momentanément pour ne pas l'aggraver et amoindrir l'actif patrimonial. Sa conversion en une procédure de redressement pour surgissement de l'état de cessation de paiement, oblige les créanciers de procéder à déclarer leurs créances auprès du syndic, cette typologie de créanciers sont désintéressés par préférence, aux autres créanciers titulaires de garanties ou sûretés art. 573 de la loi 73-17.

\subsection{A. L'éviction du dirigeant n'est plus envisageable}

Tant en droit Français ${ }^{30}$, qu'en droit Marocain, l'administration de l'entreprise est assurée par son dirigeant. En droit Marocain, il est habilité à administrer, exercer des actes de gestion seulement, il ne peut agir que sur le contrôle du syndic et du juge commissaire pour tous les actes d'exécution du plan de sauvegarde ${ }^{31}$.

En droit Français ${ }^{32}$, “ lorsque le tribunal désigne un ou plusieurs administrateurs judiciaires, il les charge ensemble ou séparément de surveiller le débiteur dans sa gestion ou de l'assister pour tous les actes de gestion ou pour certains d'entre eux".

Dans sa mission d'assistance, l'administrateur est tenu au respect des obligations légales et conventionnelles incombant au chef d'entreprise.

L'administrateur peut faire fonctionner sous sa signature les comptes bancaires ou postaux dont le débiteur est titulaire.

Tant en droit Marocain qu'en droit Français, l'ouverture de la procédure de sauvegarde n'entraine pas le dessaisissement du débiteur.

Au cours de la période de préparation de la solution, le dirigeant ne peut être dessaisi de ses prérogatives de direction de l'entreprise, le maintien en place du dirigeant est justifié, il sera anodin que le juge procède au bouleversement de la direction d'une entreprise qui n'est pas défaillante. De même, il nous paraît judicieux que le dirigeant continue à gérer l'entreprise puisqu'il a su traiter en amont les difficultés. Donc, toute initiative tendant à lui ôter cette prérogative, sera réputée inopportune, voire fâcheuse.

De surcroit, pour les deux cadres normatifs Français et Marocain, la saisine du tribunal en vue de bénéficier d'une procédure de sauvegarde est laissée à la seule initiative du débiteur, à

\footnotetext{
${ }^{30}$ Art. L 622-1 du code de commerce Français.

${ }^{31}$ Ce qui signifie que le dirigeant ne dispose pas d'une totale liberté pour gérer l'entreprise, car derrière le dirigeant, se cache en réalité le débiteur qui bénéficie d'une protection patrimoniale depuis l'ouverture de la procédure.

${ }^{32}$ Art. L 622-1 du code de commerce Français.
} 
l'exclusion des créanciers ou de toute personne intéressée. C'est une procédure volontaire, préventive, qui vise l'empêchement de la prolifération des difficultés, comme elle tend à la prévention d'une cessation de paiement imminente.

La loi Marocaine et Française sont unanimes quant à l'obligation pour le débiteur de mettre à la disposition du syndic et du juge-commissaire une liste exhaustive des biens de l'entreprise grevés de sûretés ainsi que tout son actif et les droits qui peuvent faire objet d'action en revendication ou des actions obliques de la part de leurs titulaires.

Le débiteur est tenu de faire parvenir au tribunal des documents probatoires pour une meilleure lecture des circonstances par lesquelles passe l'entreprise en difficulté ${ }^{33}$.

L'art. 561, 4 éme alinéa dispose que le chef d'entreprise peut fournir tout autre document qui montre clairement la nature des difficultés qu'éprouve l'activité de l'entreprise. La loi est très regardante sur les documents fournis, car via ces pièces justificatives le juge pourra trancher sur le caractère opportun on non d'une ouverture de la sauvegarde.

Même si le dessaisissement du débiteur n'est pas prévu, ce dernier doit se contenter d'accomplir les actes de gestion seulement, pour les autres actes étrangers à la gestion courante de l'entreprise, l'assentiment préalable du juge-commissaire est une condition incontournable pour leur validité.

A titre de comparaison avec le droit Français, on peut déduire que la loi est toujours lapidaire, les dispositions ont un sens large, indéfini, disparate et restent soumises à la technique d'analogie et d'extrapolation sur la procédure de redressement, à laquelle il faut faire référence à chaque fois. Alors que ce nouveau procédé avait sa particularité, et la sagesse voudrait qu'il lui soit réservé plus de règles réglementant la matière ${ }^{34}$.

L'assimilation entre la sauvegarde et le redressement est anodine, car si la première est préventive, précontentieuse, la seconde est purement judiciaire.

Afin de mieux cerner les missions dévolues au débiteur pendant la procédure de sauvegarde, il nous est paru judicieux de faire référence aux dispositions législatives françaises, qui offrent une vision détaillée et claire sur son rôle et de quoi il est légalement investi.

\footnotetext{
${ }^{33}$ Art. 577 du code de commerce Marocain. Livre V relatif aux difficultés des entreprises.

${ }^{34}$ Après plus de deux ans, aucun texte réglementaire n'a été prévu pour la loi 73-17, pareil pour la loi 15-95 tombée en désuétude. Cette réticence réglementaire va générer des problèmes quant à l'assimilation et l'application exhaustive de la loi.
} 
En droit Français, et pendant la période d'observation ${ }^{35}$, le président du tribunal peut ordonner toute mesure conservatoire utile à l'égard des biens du défendeur à l'action paulienne, à la demande de l'administrateur, du mandataire judiciaire, du ministère public ou d'office. Il s'agit de l'hypothèse où le débiteur a agi en fraude aux droits des créanciers, et que ces derniers pour ne pas être lésés tendaient à rendre inopposables les actes conclus en fraude à leurs droits en invoquant la fraude paulienne qui tendait à affaiblir l'actif patrimoine du débiteur pour s'échapper au désintéressement des créanciers agissant ainsi en connivence avec un acquéreur de mauvaise foi.

Toujours en droit Français, la loi confère au débiteur le droit de demander une prorogation de la période d'observation, chose qui n'est pas prévue en doit marocain, et qui reste monopolisée par le syndic habilité seul à demander le renouvellement du délai ${ }^{36}$.

Le débiteur peut proposer le nom d'un ou plusieurs administrateurs; le tribunal sollicite les observations du débiteur quand le ministère public propose la nomination d'un ou plusieurs administrateurs judiciaires. Le débiteur peut demander au juge-commissaire de saisir le tribunal aux fins de remplacer l'administrateur judiciaire.

\subsection{B. Le procédé de déclaration des créances: un passage obligé?}

L'art. 719 dispose que sont tenus de déclarer leur créance au syndic tous les créanciers dont la créance a son origine antérieurement ${ }^{37}$ au jugement d'ouverture, à l'exception des salariés.

Le législateur avait posé le critère de l'antériorité de la créance, il a énoncé également que la nature de la créance importe peu (chirographaires ou privilégiés), ainsi que le défaut de la preuve écrite

L 622-24 du code de commerce Français énonce : "a partir de la publication du jugement, tous les créanciers dont la créance est née antérieurement au jugement d'ouverture, à l'exception des salariés, adressent la déclaration de leurs créances au mandataire judiciaire dans des délais fixés par décret en Conseil d'Etat".

Le législateur Français mentionne clairement que dès la publication du jugement d'ouverture de la sauvegarde une déclaration des créances doit s'initier de la part de tous les créanciers toute catégorie concernée, son homologue Marocain, parle désormais de la déclaration des créances qu'en cas de conversion de la procédure de sauvegarde en redressement ou liquidation. Ce que

\footnotetext{
${ }^{35}$ On parle en droit Marocain plutôt de la notion de la période de préparation de la solution, fixée à 4 mois prorogeable une seule fois. Soit 8 mois, alors qu'en droit Français, cette période est de 6 mois renouvelable une seule fois mais peut être prorogée de 6 mois supplémentaire, soit 18 mois au total.

${ }^{36}$ Art. 595 de la loi 73-17.dernier alinéa.

${ }^{37}$ Tantôt, la loi parle du critère d'antériorité de la créance, tantôt de postériorité, ce critère commence à perdre sa portée, et devient moins pesant et non-sens.
} 
nous pouvons en déduire c'est que le jugement d'ouverture de la sauvegarde n'entraine pas obligation de déclarer la créance, car ce procédé concerne les créances nées antérieurement au jugement d'ouverture du redressement. Le quatrième alinéa de 1'art. 573 rappelle l'obligation qui pèse sur les créanciers dont la créance est née après le jugement d'ouverture de la sauvegarde de déclarer leurs créances.

L'analyse des derniers arrêts de jurisprudence relatifs à la sauvegarde permet de conclure que la déclaration des créances après l'ouverture de la sauvegarde et sa publication au Bulletin Officiel n'est pas prévue par ces arrêts ${ }^{38}$.

Jugement du tribunal de commerce de Casablanca n. ${ }^{\circ} 92$, dossier 2018-8301-59 du 12-juillet 2018 relatif à l'adoption d'un plan de sauvegarde pour l'entreprise Stroc Industrie.

Jugement du tribunal de commerce de Casablanca n. ${ }^{\circ} 123$, dossier 20148-8315-113, du $1^{\text {er }}$ octobre 2018, relatif à l'adoption d'un plan de sauvegarde pour l'entreprise Sotravo.

Dans les deux législations respectives, la loi parle de l'obligation de déclarer la créance dans les délais impartis par le législateur sous peine d'être forclos, et ce à partir de l'ouverture de la procédure de sauvegarde. Or, on s'interroge s'il y a obligation de déclarer la créance, pourquoi le législateur a omis de le signaler clairement dans le chapitre réservé à la sauvegarde, obligeant ainsi tout intéressé de recourir infra pour appréhender ce procédé ?

On se demande aussi, pourquoi le législateur a failli préciser de quel jugement d'ouverture s'agit-il (sauvegarde-redressement-liquidation). Doit-on recourir à l'analogie sur le redressement pour circonscrire les limites de cette nouvelle procédure, pourquoi la loi ; comme à l'accoutumée ; s'exprime toujours en filigrane?

La déclaration des créances pendant le redressement est régi par cinq art.s (art. 719 à 723) de la loi 73-17. Pendant la procédure de sauvegarde, à notre avis, il aurait été préférable de traiter le procédé de la déclaration des créances dans le chapitre réservé à la sauvegarde à l'instar de ce qui est prévu en droit Français, évitant ainsi une errance dans les dispositions législatives qui deviennent disparates.

La conversion de la sauvegarde en une procédure de redressement ou liquidation judiciaire entraine impérativement l'obligation pour les créanciers chirographaires, ou détenteurs d'une garantie de paiement (sûreté réelle) ou d'une caution (sûreté personnelle) de déclarer leurs

\footnotetext{
${ }^{38}$ Jugement du tribunal de commerce de Casablanca n. ${ }^{\circ}$ 92, dossier 2018-8301-59 du 12-juillet 2018 relatif à l'adoption d'un plan de sauvegarde pour l'entreprise Stroc Industrie. Jugement du tribunal de commerce de Casablanca n. ${ }^{\circ} 123$, dossier 20148-8315-113, du $1^{\text {er }}$ octobre 2018, relatif à l'adoption d'un plan de sauvegarde pour l'entreprise Sotravo.
} 
créances déduction faite du montant des créances déjà perçues ${ }^{39}$. Arrêt de la cour de cassation, $\mathrm{n}^{\circ} 1024$ du 22-09-2004, dossier n. ${ }^{\circ}$ 2003-2-3-555.

La déclaration des créances s'impose également à tout créancier dont la créance a pris naissance ultérieurement à l'ouverture de la procédure de sauvegarde, et non après le redressement judiciaire qui reste exonérée de ce procédé ${ }^{40}$. Arrêt de la Cour de cassation Rabat, ch.com. $\mathrm{n}^{\circ} 1004,12-10-2005$.

\section{QUELLE ATTRACTIVITÉ POUR LES PARTENAIRES DU DÉBITEUR?}

Le constat global d'échec des procédures préventives préexistantes, nous emmène à douter de la réussite de ce nouveau mécanisme de sauvegarde, car cet insuccès ou bien ce "degré d'applicabilité faible" tient essentiellement à une profonde méconnaissance du régime par les chefs d'entreprises, ainsi qu'à des imperfections techniques qui limitent sa portée et entravent sa réussite. Il semble que la mise en œuvre de certaines modifications du texte, ainsi qu'une meilleure information et sensibilisation sur l'intérêt de la prévention, seraient de nature à dynamiser un dispositif préventif actuellement en déshérence.

Une prise de conscience précoce est indéniablement importante. La prévention reste aujourd'hui une voie peu empruntée par la plupart des juridictions. Quant aux chefs d'entreprises, leur méconnaissance ou leur méfiance à l'égard de la prévention les amène à manquer d'initiative et c'est ce qui empêche le décollage de la procédure préventive à temps utile $^{41}$.

En droit Marocain, l'attractivité pour la sauvegarde s'est manifestée via la demande introduite par l'entreprise Stroc industrie ${ }^{42}$, qui n'a pas tergiversé à consommer ce nouveau mécanisme. Elle a demandé le bénéfice de la sauvegarde, une initiative audacieuse couronnée de réussite, car l'entreprise a été soumise au bénéfice d'ouverture de la sauvegarde, puisqu'elle n'était pas en cessation de paiement, et présentait des plans sérieux de remboursement de ses dettes.

\footnotetext{
39 Si les créanciers détenteurs de sureté publiée ou un contrat de crédit -bail publiés sont avertis et avisés personnellement par le syndic de l'ouverture de la procédure, cela ne leur dispense pas de procéder à la déclaration de leurs créanciers sous peine d'être évincés et forclos. Arrêt de la cour de cassation, n 1024 du 22-09-2004.dossier $\mathrm{n}^{\circ} 2003-2-3-555$.

${ }^{40}$ Arrêt de la Cour de cassation Rabat, ch.com. $\mathrm{n}^{\circ} 1004,12-10-2005$. Revue recueil des arrêts de la cour suprême, n. ${ }^{\circ} 66$. "La déclaration des créances au syndic ne peut concerner que les créances antérieures au jugement d'ouverture".

${ }^{41} 8439$ faillites en 2019 dues à l'augmentation des impayés et à la dégradation de la conjoncture, l'avenir n'est pas prometteur pour les entreprises en 2020 il y a un risque constant de voir des entreprises de gros calibre disparaitre furtivement. Selon les indicateurs d'inforisk d'Euler Hermès, spécialiste du renseignement commercial, détenteur de l'indice global de l'évolution des défaillances d'entreprise.

42 "Stroc industrie" première entreprise ayant bénéficié du plan de sauvegarde quinquennal, quelques mois seulement après l'adoption du nouveau mécanisme de sauvegarde.
} 
Son projet de plan de sauvegarde, était admis vu la crédibilité des modalités d'apurement du passif patrimonial du débiteur, ainsi que la fiabilité des garanties fournies pour l'exécution du projet de sauvegarde.

En droit Français; Une affaire très remarquable ${ }^{43}$ et commentée, avait mis le monde des procédures collectives en émoi. Arrêt "cœur défense", CA Paris, pole 5, ch. 9, n09/ 22756. Elle valide le recours à la procédure de sauvegarde pour la holding d'acquisition Hold et Dame Luxembourg. Après moult tergiversations sur l'application exacte de la jeune procédure de sauvegarde, enfin une illustration saisissante surgira pour mettre fin aux interrogations relatives à la caractérisation des difficultés rencontrées par le demandeur. Le contentieux de l'affaire cœur défense ou "Hold", avait fourni une multitude de clarifications, ainsi que des conditions suffisantes pour juger de l'opportunité d'ouvrir une sauvegarde en faveur d'un débiteur malchanceux.

Dans cette affaire riche d'enseignements, il a été décidé trois points essentiels justifiant la demande d'ouverture d'une procédure de sauvegarde, en évitant, éventuellement, toute tentative d'instrumentalisation ou de détournement de la part du débiteur, qui procédera à des demandes un peu hâtives, alors qu'il est toujours capable de désintéresser ses créanciers et d'honorer ses dettes ${ }^{44}$.

\section{1. la sauvegarde entre emballement des uns et désengouement des autres}

Si l'adoption; à l'instar de ce qui prévu en droit Français; du mécanisme de sauvegarde est une initiative salutaire, pour le débiteur en difficulté, lui permettant de bénéficier de la règle de la seconde chance, elle est pour les créanciers moins attractive, car elle leur empêche de poursuivre leur débiteur pour être payer sur leurs créances.

Le débiteur bénéficie par conséquent de la Suspension Provisoire des Poursuites individuelles, (ci-après SPP) qui suspend toute action en justice de la part des créanciers, comme elle interdit aussi au débiteur de procéder au règlement d'un créancier au détriment d'un autre.

Si la sauvegarde est bienveillante pour les uns, elle ne l'est pas pour les autres, cela constitue l'une des contraintes du droit des procédures collectives, qui parfois parait insusceptible de conjuguer tous les impératifs de façon simultanée, et satisfaire tous les partenaires du débiteur, ou bien les parties prenantes.

\footnotetext{
${ }^{43}$ Arrêt "cœur défense", CA Paris, pole 5, ch. 9, n09/ 22756, actualité des procédures collectives civiles et commerciales, $\mathrm{n}^{\circ} 7,15$ avril 2011.

${ }^{44}$ PETEL.P., "les nouveaux critères d'ouverture de la sauvegarde", Revue droit et patrimoine, décembre 2009, p. 59. L'arrêt "cour de défense" offre un exemple attendu de l'exercice du pouvoir d'appréciation laissé au juge du fond dans l'appréciation du critère d'ouverture de la sauvegarde, car celui-ci est défini d'une manière très souple, spécialement depuis l'ordonnance du 18 décembre 2008.
} 


\subsection{A. La constitution de comités des créanciers : une méthode de concertation efficiente?}

La procédure de sauvegarde des entreprises, en accordant un droit au débiteur, a voulu équilibrer la balance en renforçant les droits des créanciers tout en leur permettant de s'organiser sous forme de comités des créanciers.

Ce dispositif se traduit par une meilleure participation des créanciers du moins pour ceux qui font partie de ces comités. Le législateur Marocain ${ }^{45}$ à travers l'art. 606 octroie à ces créanciers un pouvoir et une influence déterminants sur l'issue de la procédure à l'instar de ce qui est prévu par son homologue Français qui permet à son tour aux créanciers de se constituer en comité $^{46}$. art.s L626-29 à L626-35 du code de commerce français.

La constitution de comité est conditionnée en droit Français par des seuils. Le législateur a voulu retenir un critère objectif, selon lequel, les débiteurs dont les comptes ont été certifiés par un commissaire aux comptes ou établis par un expert-comptable et dont le nombre des salariés est supérieur à 150, ou le chiffre d'affaire dépasse à son tour les 20 millions d'euros, peuvent constituer des "comités de créanciers".

On constate que la constitution de comité n'est possible que si on est en présence d'une grande entreprise. Notons, également, que ces seuils ne sont pas cumulatifs, ils sont alternatifs.

Le droit Français, dans l'art. L626-29 et suivant du code de commerce, prévoit la constitution de deux comités : le comité des établissements de crédit et le comité des principaux fournisseurs de biens ou de services.

La distinction entre créanciers privilégiés et créanciers chirographaires a été rejetée, même si le comité des établissements de crédit est en réalité composé de créanciers titulaires de suretés ou de garanties, tandis que les membres du comité des fournisseurs sont en général des créanciers chirographaires. Les créanciers visés sont uniquement les établissements de crédit et les fournisseurs, à l'exclusion des créanciers salariaux, qui bénéficient toujours d'un traitement favorable.

Le droit Marocain, quand il a prévu la création des assemblés des créanciers avait omis de faire allusions à leur collaboration éventuellement lors de l'adoption d'un plan de sauvegarde. La loi prévoit, dans l'art. 606 du code de commerce "lors de l'ouverture de la procédure de redressement judiciaire, une assemblée des créanciers doit être constituée..."

\footnotetext{
${ }^{45}$ Selon les termes de l'art. 606 code de commerce Marocain, "l'entreprise doit avoir un commissaire aux comptes, elle réalise un chiffre d'affaire annuel de 25 millions de dirhams et embauche plus de 25 salariés".

${ }^{46}$ En droit Français, on parle de "comité des créanciers" qui ne date pas d'hier comme c'est le cas pour le droit marocain. Le comité des créanciers était instauré par la loi de sauvegarde des difficultés de l'entreprise du 26 juillet 2005. En droit Marocain, "l'assemblée des créanciers" était récemment prévue par la nouvelle loi 73-17 relative aux difficultés de l'entreprise.
} 
Le droit Marocain, contrairement à son homologue Français, n'a pas désigné la forme de ces assemblées, ni leur typologie.

En droit Français, Ces comités prennent les formes suivantes et ils sont au nombre de deux: le premier comité est constitué des établissements de crédit (banques, sociétés financières...). Notons à ce point que l'ordonnance du 18 décembre 2008 classe les établissements assimilés aux établissements de crédit, dans cette catégorie. Elle parle désormais de toute entité ayant conclu avec le débiteur une opération de crédit sera membre du comité des établissements de crédit. Le second comité est constitué des principaux fournisseurs de biens ou services à l'entreprise.

\subsection{B. La préservation du mécanisme de suspension provisoire des poursuites individuelles}

Le législateur Marocain prévoyait dans l'art. 686 du code de commerce que "le jugement d'ouverture suspend ou interdit toute action en justice de la part de tous les créanciers dont la créance a son origine antérieurement au dit jugement..."47.

Les créanciers, inquiets par la situation de l'entreprise débitrice et soucieux de récupérer leur dû avant son aggravation, sont tentés d'exercer des poursuites dans ce but. Mais, ces poursuites et procédures d'exécutions constituent des pressions incompatibles avec l'esprit de la sauvegarde.

Réciproquement et par équilibre des concessions des parties, la même ordonnance interdit au débiteur, sauf autorisation spéciale du juge-commissaire et sous peine de nullité, de payer en tout ou en partie, une créance quelconque née antérieurement à la date du prononcé de la décision de suspension pour retirer une chose primordiale pour la poursuite de l'activité de l'entreprise.

Le bénéfice de cette dérogation aux principes généraux de la Suspension provisoire des poursuites (ci-après SPP) profite aussi au créancier titulaire d'une sûreté qui peut à son tour demander au juge-commissaire la vente du bien mobilier quand ce dernier présente des signes de dépérissement imminent. Dernier alinéa de l'art. 686 du code de commerce Marocain.

En droit Français, le texte est sans équivoque : art. L622-7 du code de commerce qui traite la procédure de sauvegarde dispose: "Le jugement ouvrant la procédure emporte, de plein droit, interdiction de payer toute créance née antérieurement au jugement d'ouverture, à l'exception du paiement par compensation de créances connexes. Il emporte également, de plein droit,

\footnotetext{
${ }^{47}$ A préciser dans ce sens que la prononciation de la Suspension Provisoire des poursuites individuelles arrête également l'accumulation des cours des intérêts, dès le jugement d'ouverture. Cependant, ces intérêts deviennent cumulables dès l'adoption du plan de sauvegarde. Voir art. 692 et 693 du code de commerce Marocain.
} 
interdiction de payer toute créance née après le jugement d'ouverture, Ces interdictions ne sont pas applicables au paiement des créances alimentaires".

L'ordonnance prise par le président, pour la suspension des poursuites et exécutions, interdit et suspend les poursuites et les voies d'exécutions des créanciers dont les titres sont antérieurs à la date de son prononcé. Les poursuites et voies d'exécutions touchées concernent, les meubles et les immeubles du débiteur et qui tendent à sa condamnation au paiement d'une somme d'argent, ou à la résolution d'un contrat pour défaut de paiement d'une somme d'argent.

L'interdiction de paiement s'étend au désintéressement des cautions, qui acquitteraient des créances également antérieures à l'ordonnance. Elle embrasse enfin, tout acte de disposition étranger à la gestion normale de l'entreprise. En somme, elle touche tous les actes de nature à diminuer le gage des créanciers, à favoriser ou défavoriser certains d'entre eux.

En effet, en plus de l'interdiction des paiements, elle empêche le débiteur de procéder à tout acte de disposition étranger à la gestion normale ou courante ${ }^{48}$. Il ne peut plus alourdir ses charges, notamment, par l'aliénation des immeubles et des équipements, ni par la constitution des sûretés, hypothèques ou nantissement ${ }^{49}$.

Pis encore, l'interdiction des paiements est sévèrement étendue, car le débiteur ne peut même plus désintéresser les cautions qui auraient acquitté des créances antérieures à l'ordonnance. Cette dernière interdiction aggrave les engagements des cautions ; non seulement les créanciers de l'entreprise en difficulté, ne peuvent leur demander d'exécuter le cautionnement, malgré l'ouverture de la procédure de sauvegarde, mais de plus ils ne peuvent pas exercer leurs recours contre le débiteur pendant toute la durée de la suspension. Cette solution semble particulièrement sévère pour les banques qui se portent caution, mais elle leur profite de la même manière quand elles sont bénéficiaires du cautionnement.

La finalité ultime de cette mesure, était de faire bénéficier l'entreprise en difficulté d'un moment de répit, face à la pression de ses divers créanciers. Elle permet également de dompter les créanciers récalcitrants, qui profitent de la situation pour engager des actions en exécution forcée, compromettant ainsi une négociation utile à l'intérêt général par des initiatives intempestives.

Evoquer la SPP nous emmène inexorablement à traiter le sort de la caution . Quid de la personne qui cautionne le débiteur?

\footnotetext{
${ }^{48}$ Exception, Le juge-commissaire peut autoriser le débiteur à faire un acte de disposition étranger à la gestion courante de l'entreprise, à consentir une hypothèque, un gage ou un nantissement ou à compromettre ou transiger (art. L 622-7 du code de commerce Français).

49 Idem pour le droit Marocain, selon l'art. 699, le débiteur ne peut plus grever un bien d'hypothèque, de nantissement, ou de privilèges postérieurement au jugement d'ouverture de la sauvegarde.
} 
- La SPP interdit au débiteur de payer la caution qui acquittait une créance née avant le jugement d'ouverture de la sauvegarde ;

- La SPP interdit aux créanciers du débiteur de recourir contre la caution ${ }^{50}$. Cass.ch. com. 30 janvier $2019 \mathrm{n}^{\circ}$ de pourvoi .16-18468, pour obtenir le paiement de leurs créances, car la caution peut se prévaloir de l'adoption du plan de sauvegarde selon les termes de 1 'art. $572^{51}$, le cours des intérêts de retard et majorations ne se cumulent plus, ils sont en instance également dès l'ouverture de la sauvegarde. Par contre, ils deviennent cumulables dès l'adoption du plan de sauvegarde art. 693 du code de commerce Marocain ${ }^{52}$.

- La caution quant à elle ne peut pas recourir contre le débiteur dès la prononciation du jugement d'ouverture de la sauvegarde, soit pendant toute la période de la préparation de la solution.

Sont inopposables à la caution personne physique, les créances non déclarées au passif du débiteur art. 695 dernier alinéa du code de commerce Marocain. Donc, la caution peut se prévaloir du non déclaration de la créance par le créancier ${ }^{53}$.

\subsection{La qualification juridique du nouveau mécanisme de sauvegarde}

La sauvegarde présente des incertitudes qui persistent toujours sur son vrai caractère. Tantôt, on croit à son caractère collectif, tantôt on la classe parmi les procédures amiables.

Pour faire dissiper tous ces doutes qui entourent cet arsenal attractif et dissuasif, il est temps de projeter la lumière sur ses caractéristiques, en analysant le pour et le contre et on dégageant, à la fin, une réponse claire sur sa nature pour ne pas induire en erreur ceux qui veulent ou souhaitent y recourir. Cette nouvelle pierre apportée à la construction du droit des entreprises en difficulté qui se bâtit soigneusement est riche d'enseignements.

Nous commencerons par lever le voile sur le caractère véridique de cette procédure, est-t-elle hybride, semi judiciaire, segmentée? Une qualification s'impose donc face à la multiplication

\footnotetext{
${ }^{50}$ On se réfère à la caution personne physique. Quant à la caution personne morale, elle est tenue de la dette échue au jour de l'ouverture de la sauvegarde. Cass.ch. com. 30 janvier $2019 \mathrm{n}^{\circ}$ de pourvoi .16-18468.

${ }^{51}$ Art. 695 du code de commerce Marocain, apporte à son tour des précisions concluantes, quand il empêchait les créanciers de recourir contre la caution et coobligés. Si la résolution est heureuse pour la caution, elle ne l'est pas pour les créanciers qui se trouvent devant une impasse.

${ }^{52}$ C. Cass, ch.com, 17 avril 2019. Pourvoi n ${ }^{\circ}$ 17-19-555. Selon cet Arrêt de la cour de cassation Française, le créancier ne peut pas réclamer à la caution personne physique le paiement d'intérêt ou majorations postérieurement au jugement d'ouverture de la sauvegarde, toute mesure contraire ne pourra que violer l'art. L622-28 du code de commerce Français.

53 L'absence de déclaration de créance n'entraîne pas extinction de la créance mais uniquement son inopposabilité à la procédure collective.
} 
des dénominations fournies par les professionnels et les ténors du droit des procédures collectives.

\subsection{A. La sauvegarde: une procédure amiable, collective ou hybride?}

Evitant tout pléonasme, car c'est déjà signalé à maintes reprises, l'ouverture au bénéfice d'une procédure de sauvegarde n'est possible que si le débiteur n'est pas acculé déjà à la cessation de paiement, sinon un redressement judiciaire doit être ouvert.

Or, le débiteur qui arrive à prouver que des perspectives s'annoncent à l'horizon, et qui laissent présager la survenance d'un état de cessation de paiement peut justifier l'ouverture de la sauvegarde ${ }^{54}$. Ce tiraillement entre absence de cessation de paiement et le risque imminent de surgissement de cet état est source d'amalgame en droit Marocain.

En droit Français ce pronostic d'impécuniosité fut écarté, on se contente d'évoquer l'existence des difficultés.

Autres justificatif, le déroulement de la procédure de sauvegarde sous la houlette du tribunal de commerce, avec la collaboration de l'autorité judiciaire (juge-commissaire, président du tribunal de commerce et syndic) donne une coloration juridique à ce mécanisme de sauvegarde, car le juge intervient pour dire le droit, le ministère public quant à lui est là pour poursuivre et faire prévaloir les intérêts de l'entreprise et de tout le tissu économique national.

L'autorité judicaire n'est pas une simple magistrature économique qui exerce une sorte de pression psychique, comme c'est le cas pour l'ancien règlement amiable ${ }^{55}$, ou l'actuelle procédure de conciliation où le président du tribunal de commerce joue un rôle de catalyseur pour inciter les parties au respect des engagements respectivement convenus entre eux. Pendant l'alerte externe, ou la conciliation, on assiste plutôt à un juge dénué de ses droits naturels de dire le droit et de prononcer des sanctions.

Lors de la sauvegarde, le tribunal reçoit le chef d'entreprises en chambre de conseil, les négociations et les explications se déroulent à huit clos.

Le caractère volontaire et non pas obligatoire de la demande d'ouverture de la sauvegarde, reflète également que le débiteur ; de son propre gré, peut exiger le bénéfice de la sauvegarde. La loi s'est contentée par contre de tracer les contours de cette demande pour éviter un usage galvaudé de ce mécanisme.

\footnotetext{
${ }^{54}$ Art. 561 de la loi 73-17 du code de commerce Marocain.

${ }^{55}$ Ce vocable de "règlement amiable" était prévu par l'ancienne loi 15-95 abrogée, il était substitué par le concept de "conciliation" prévu par la nouvelle loi 73-17 dans les art.s 551 à 559 du code de commerce Marocain.
} 
La procédure de sauvegarde appréhende l'ensemble des biens du débiteur ${ }^{56}$ et contrait l'ensemble des créanciers, même si certains ${ }^{57}$ de par la nature de leur créance échappent de façon justifiée à la main mise de la procédure ${ }^{58}$. d'autres par contre ; pour être désintéressés ; doivent être soumis à un gel du passif patrimonial, ils leur est interdit d'agir individuellement en justice car cela ne peut être que contraire à l'esprit du mécanisme de sauvegarde, et du droit des procédures collectives en général qui tend essentiellement à offrir des moratoires de paiement.

\subsection{B. Le caractère "ambivalent" de la nouvelle procédure de sauvegarde}

Une simple lecture en diagonale des dispositions législatives régissant le mécanisme de sauvegarde peut révéler le caractère mitigé o ambigu de cette procédure.

Les professionnels de droit, les académiciens et les praticiens tergiversent toujours sur son vrai caractère ou sa vraie qualification juridique, puisqu'il s'agissait d'une procédure qui se trouve à mi-chemin entre l'amiable et le contentieux, on peut la caser dans la phase de préinsolvabilité, car le débiteur ne doit pas être en cessation de paiement.

Ce raisonnement peut rapidement être "chambardé", car si les procédures amiables sont caractérisées par la discrétion et la confidentialité, la sauvegarde ne l'est pas. Il suffit de faire recours aux droits des tiers qui doivent être informés si leur interlocuteur a bénéficié d'un plan de sauvegarde ou de restructuration. Si le traitement amiable des difficultés est placé sous le signe de la nécessaire discrétion pour préserver la renommée du débiteur, le traitement judiciaire est, en revanche, public et ne peut être collectif qu'à la condition d'être entouré de la plus large publicité.

Le jugement prononçant la sauvegarde ne peut échapper aux procédures de publicité telles quelles sont bien définies par le code de commerce ${ }^{59}$. Un anéantissement de la règle de

\footnotetext{
${ }^{56}$ Selon l'art. 1241 du droit des obligations et des contrats (ci-après DOC): "les biens du débiteur sont le gage commun des créanciers". Même l'action paulienne qui est une voie de droit commun, demeure régie sur la base de cet art., elle offre à tout créancier lésé d'attaquer les actes effectués par son débiteur en fraude à ses droits devant la justice.

Le patrimoine d'affection qui le seul peut être soumis à la procédure collective, à l'exception du patrimoine personnel, ne trouve pas application en droit marocain, contrairement au droit français, où la confusion du patrimoine fut écartée, pour ne pas permettre aux procédures collectives d'engloutir le patrimoine personnel du débiteur malchanceux.

${ }^{57}$ Art. 711 du code de commerce Marocain précisait que les biens appartenant au conjoint du débiteur soumis à la procédure de sauvegarde, ainsi que les biens de ses enfants mineurs peuvent être pris en compte dans l'évaluation de l'actif patrimonial du débiteur. Il revient au syndic de prouver leur origine pour être réunis à l'actif.
}

${ }^{58}$ LYAZAMI, N., "le rang de paiement des créanciers dans les procédures collectives: comment trouver un dosage subtil à ce dilemme?", Revue juridique Censeur, n. ${ }^{\circ}$, Avril 2019. Le super privilège des salariés, dont la nature de la créance est alimentaire, les créanciers postérieurs au Jugement d'ouverture de la sauvegarde aussi.

${ }^{59}$ Art. 584 de la loi 73-17 du code commerce Marocain. 
confidentialité est possible cette fois, pendant la procédure de sauvegarde. Et si la confidentialité est maintenue lors de la conclusion de l'accord de conciliation, elle est clairement déclinée lors de la publication du jugement d'ouverture de la sauvegarde.

Art. 584 dispose: "Le jugement d'ouverture de la procédure prend effet à partir de sa date. Il est mentionné sans délai aux registres du commerce local et central.

Dans les huit jours de la date du jugement, un avis de la décision comportant la dénomination de l'entreprise telle qu'elle figure au registre de commerce et son numéro d'immatriculation audit registre, est publié par le greffier dans un journal d'annonces légales et au "Bulletin officiel”. Il invite les créanciers à déclarer leurs créances au syndic désigné. Cet avis est affiché au panneau réservé à cet effet au tribunal immédiatement après que ce dernier prononce le jugement.

Le jugement doit être mentionné sur les livres de la conservation foncière, les registres d'immatriculation des navires et aéronefs et les autres registres assimilés, selon le cas.

Dans le délai de huit jours, le jugement est notifié au chef de l'entreprise et au syndic par les soins du greffier".

En droit Français, La lecture de 1'art. $425,2^{\circ}$ du code procédure civile Français laisse comprendre qu'il doit y avoir communication des procédures de sauvegarde au ministère public, (ci-après MP) cette communication a été qualifiée de -obligatoire- . Ce texte de base n'est pas le seul à conférer ce droit au MP, garant de l'ordre public économique.

Même le livre VI du code de commerce Français prévoit la communication des jugements de tribunal relatifs à l'ouverture de la procédure de sauvegarde au MP. Certes des dispositions similaires faisaient défaut en droit Marocain, et donc les jugements relatifs aux procédures de sauvegarde ne sont pas communiqués au $\mathrm{MP}^{60}$.

Mais il faut mettre en exergue le cas d'extension de la procédure de sauvegarde qui peut être déclenchée par le MP pour confusion de patrimoine ou fictivité.

A notre humble avis, donner une qualification pointilleuse au nouveau mécanisme de sauvegarde, peut paraitre prétentieux, dans la mesure où un débat houleux subsiste toujours sur l'éventuelle ouverture de cette procédure au profit des dirigeants d'entreprises se trouvant déjà sombrés dans la cessation de paiement, sous condition de prouver que cette insolvabilité était intrinsèquement liée à la conjoncture actuelle relative à la pandémie mondiale du Covid 19 .

\footnotetext{
${ }^{60}$ LYAZAMI, N., "Éléments de réflexion sur rôle du Ministère Public dans les procédures d'insolvabilité : étude analytique, critique et comparative entre le droit marocain et le droit Français" Revue Marocaine d'administration locale et de développement, $\mathrm{n}^{\circ} 150-151$, janvier-avril 2020, p. 63 et suiv.
} 
Nonobstant les doutes qui planent toujours sur sa nature, on ne peut pas nier que la procédure de sauvegarde est un mécanisme de sauvetage des entreprises qui présentent de fortes chances de rebondissement et de renflouement, puisqu'elles sont toujours in bonis.

par conséquent, c'est une procédure préventive, anticipative visant à éradiquer le mal le plus en amont possible pour conjurer les conséquences calamiteuses résultantes du dépôt de bilan des entreprises sur la santé financière de tout le tissu économique du Royaume du Maroc, mais aussi sur la santé psychique et mentale ${ }^{61}$ des chefs d'entreprise aux abois .

\section{EPILOGUE}

Nous avons mis en relief la procédure de sauvegarde et sa variante SFA, en démontrant son extranéité en droit Marocain. Nous avons voulu démontrer comment l'application de cette institution en droit Marocain va générer des problèmes en termes de son application et de sa généralisation. Vu le caractère lapidaire et opaque de certains articles ${ }^{62}$.

Les créanciers sont appelés de plus en plus à se rallier à la prévention, une voie qui ne peut qu'être bénéfique pour le sort de leurs créances.

Pour préserver des entreprises saines, il ne faut pas compter seulement sur les initiatives du législateur, un effort commun est à déployer. Tous les acteurs de la prévention sont appelés à participer activement et efficacement au bon déroulement de la procédure de prévention.

La réussite de la prévention passe essentiellement par la promptitude de la réaction du chef d'entreprise pour éviter la cessation de paiement souvent fatale, mais également une prise de conscience et le bon réflexe seront idoines.

Nous estimons primordiale que la transposition d'une institution nouvelle pour peaufiner le cadre normatif existant ne doive pas être faite à l'aveuglette, sans que les circonstances ne soient bien cernées et circonscrites avec circonspection, pour éviter de tomber dans les mêmes obstacles dus à l'application tout court du droit Français et aux antinomies auxquelles les juges se trouvent confrontées. On peut s'inspirer mais sans tomber dans l'excès des prétentions.

Et in fine, pour clore nos propos, nous estimons que la concertation, la négociation, et l'envie incessante de parvenir à réduire à néant tous les conflits, restent les voies les plus privilégiées pour sauver l'entreprise et éviter les conséquences chaotiques que peut engendrer sa disparition.

\footnotetext{
${ }^{61}$ LYAZAMI, N., "Pandémie mondiale du Covid19, traumatisme psychologique des dirigeants d'entreprise en difficulté : quelle capacité de résilience?", L'Institut Marocain de l'Information Scientifique et Technique (IMIST), Revue Journal of Integrated Studies in Economics, Law, Technical Sciences \&Communication, n. ${ }^{\circ} 1$ novembre 2020.

${ }^{62}$ A noter que le législateur Marocain n’a pas encore prévu des textes réglementaires pour la nouvelle loi 73-17.
} 


\section{BIBLIOGRAPHIE}

ALAMI MACHICHI DRISSI, M., Droit des affaires, Dar Al Kalam, 2006.

CHOUKRI SBAI, A., Les procédures de prévention et de traitement des difficultés des entreprises, MDC, Rabat 2000.

EL HAMMOUMI, A., Le droit des difficultés des entreprises : la prévention des Difficultés, redressement et liquidation, Volume 1, 2006.

LAHBIB RHALIB, M.M., Entreprises en difficulté: quels sont vos droits, La croisée des chemins, 2016.

LE CORRE, P.M et LE CORRE BROLY. E., Droit des entreprises en difficulté, 2ème édition Sirey, 2006.

LE CORRE, P.M., Droit des entreprises en difficulté, 4ème édition, Dalloz. 2011.

LFROUJI, A., La cessation des paiements dans le droit des entreprises en difficulté, Ideguel, Rabat, 2005. (en árabe).

LIENHARD, A., Procédures collectives : prévention, conciliation, sauvegarde financière accélérée, 4ième édition Delmas, 2011-2012.

LYAZAMI, N., "La prévention des difficultés de l'entreprise: approche comparative entre le Droit Marocain et le Droit Français", Thèse doctorale, université du Sud-Toulon Var. France, 4 juin 2013.

LYAZAMI, N., "Le contentieux du chèque et la préservation d'une entreprise viable : quelle corrélation”. Revue du droit Marocain.n. ${ }^{\circ} 38$, juillet, 2018.

LYAZAMI, N., "Plus de vingt ans d'application du droit préventif de difficulté de l'entreprise : un bilan mitigé", Revue Marocaine d'administration locale et de développement, n. ${ }^{\circ} 148$, septembre-octobre, 2019.

LYAZAMI, N., "Le rang de paiement des créanciers dans les procédures collectives : comment trouver un dosage subtil à ce dilemme?”. Revue juridique Censeur.nº 7, Avril, 2019.

LYAZAMI, N., "Éléments de réflexion sur rôle du Ministère Public dans les procédures d'insolvabilité: étude analytique, critique et comparative entre le droit marocain et le droit Français", Revue Marocaine d'administration locale et de développement, $\mathrm{n} .{ }^{\circ} 150$ 151, janvier-avril, 2020.

LYAZAMI, N., "Pandémie mondiale du COVID19, traumatisme psychologique des dirigeants d'entreprise en difficulté: quelle capacité de résilience?", L'Institut Marocain de 
l'Information Scientifique et Technique (IMIST), Revue Journal of Integrated Studies in Economics, Law, Technical Sciences \&Communication. $n^{\circ} 1$ novembre, 2020.

SQUALLI, A., Droit et pratique en matière de la faillite et de liquidation judiciaire des entreprises, 1ére édition, 1995.

TAK TAK, H., "La procédure de sauvegarde des entreprises en difficulté : un modèle français ou un modèle américain?", Revue marocaine de droit, d'économie et de gestion.n ${ }^{\circ} 53$ ed., 2008.

VIDAL, D., "Droit des entreprises en difficulté : prévention, conciliation, sauvegarde", n. ${ }^{\circ} 3$ ed., L'extenso, 2010.

XAVIER LUCAS, F et L'ECUYER, H., La réforme des procédures collectives, la Loi de sauvegarde article per article, LGDJ, 2008. 